\title{
Future of Automated Insulin Delivery Systems
}

\author{
Jessica R. Castle, MD, J. Hans DeVries, MD² and Boris Kovatchev, $\mathrm{PhD}^{3}$
}

\begin{abstract}
Advances in continuous glucose monitoring (CGM) have brought on a paradigm shift in the management of type 1 diabetes. These advances have enabled the automation of insulin delivery, where an algorithm determines the insulin delivery rate in response to the CGM values. There are multiple automated insulin delivery (AID) systems in development. A system that automates basal insulin delivery has already received Food and Drug Administration approval, and more systems are likely to follow. As the field of AID matures, future systems may incorporate additional hormones and/or multiple inputs, such as activity level. All AID systems are impacted by CGM accuracy and future CGM devices must be shown to be sufficiently accurate to be safely incorporated into AID. In this article, we summarize recent achievements in AID development, with a special emphasis on CGM sensor performance, and discuss the future of AID systems from the point of view of their input-output characteristics, form factor, and adaptability.
\end{abstract}

Keywords: Artificial pancreas, Continuous glucose monitoring, Type 1 diabetes.

\section{Introduction}

A DVANCES IN CONTINUOUS GLUCOSE MONITORING (CGM) technology have brought in the era of automated insulin delivery (AID). AID involves CGM values feeding into a closedloop control (CLC) algorithm running on an insulin pump (embedded AID) or on a smart phone (Mobile AID). Advanced CLC algorithms typically use CGM data, feedback from insulin delivery (e.g., insulin-on-board, or IOB, calculation), and potentially other signals to modify the insulin delivery rate. Due to its promise to optimize diabetes control, AID may soon emerge as the standard of care for managing type 1 diabetes.

One challenge of managing diabetes without significant hypoglycemia relates to the wide fluctuation in insulin requirements to maintain euglycemia, between people and for the same person from day-to-day and in different situations. Insulin needs are impacted by a host of factors, including food intake, activity level, illness, emotional stress, sleep deprivation, and menstrual cycle. ${ }^{1}$ A second challenge is the time delay between subcutaneous insulin injection and insulin action to lower blood glucose levels, which can extend to more than an hour. ${ }^{2,3}$ As a result, it is exceedingly difficult for an individual to accurately predict how much insulin is needed in any particular situation, often resulting in hypo- or hyperglycemia.

One solution to this dilemma is to automate insulin delivery such that the CLC algorithm responds to changes in glucose levels by appropriately adjusting insulin delivery and taking into account the variation of insulin demands for each person, at any point in time. A fully automated adaptable AID system still does not exist; however, advances are made to improve system inputs (e.g., CGM accuracy and others), CLC algorithms (e.g., adaptive model-predictive control and realtime estimation of a person's state), and system outputs, for example, insulin alone, or delivery of multiple hormones such as insulin+glucagon or insulin+synthetic amylin. In this article we summarize recent achievements in AID development, with a special emphasis on CGM sensor performance, and discuss the future of AID systems from the point of view of their input-output characteristics, form factor, and adaptability. The topic of CLC algorithms has been reviewed elsewhere. ${ }^{4-6}$

\section{The Importance of CGM Accuracy}

The quality of the input signal is essential for any control system. The primary input for the AID systems is the continuous stream of glucose values provided by a CGM. How accurate does a CGM need to be to enable safe and effective functioning of an AP system? Beyond the obvious "the more accurate the better" lies the question whether we can define a threshold in terms of mean absolute relative difference (MARD) that if met, guarantees more or less safe operation of an artificial pancreas system. CGM accuracy has improved

\footnotetext{
${ }^{1}$ Division of Endocrinology, Harold Schnitzer Diabetes Health Center, Oregon Health \& Science University, Portland, Oregon.

${ }^{2}$ Department of Endocrinology, Academic Medical Center, University of Amsterdam, Amsterdam, The Netherlands.

${ }^{3}$ Center for Diabetes Technology, University of Virginia, Charlottesville, Virginia.
} 
over the years. ${ }^{7,8}$ This is in part due to factors that remain undisclosed by manufacturers and in part due to factors that have been made public. These include improvements in CGM signal filtering, denoising, and enhancement that resulted in significantly better sensor performance. ${ }^{9}$ Furthermore, reduction of biofouling and enzyme degradation can reduce signal error and improve reliability and longevity of CGM, ${ }^{10,11}$ as well as standardized and less traumatic sensor insertion. ${ }^{10}$

Although a consensus guideline was proposed in $2008,{ }^{12}$ the scientific community has yet to agree upon how to best assess CGM accuracy. Metrics for accuracy include MARD, mean absolute difference, which is commonly used for assessment of the hypoglycemic range, percentage of egregious errors ( $>20 \%$ error), Clarke error grid, ${ }^{13}$ continuous glucose-error grid, ${ }^{14}$ Parkes error grid, ${ }^{15}$ and the Surveillance error grid. ${ }^{16}$ The correct reference further complicates the issue. Unlike the assessment of blood glucose meter accuracy using split samples using ISO 15197, the direct measurement of interstitial glucose is not readily available. Often venous blood is used and measured by YSI as the gold standard, although one should note that the use of capillary blood glucose may result in a lower MARD. ${ }^{17}$ Realworld use scenarios may introduce additional sources of inaccuracy, including pressure-induced signal attenuation, which can cause an artificially low glucose reading, including at night when someone is sleeping, and interfering substances. ${ }^{18,19}$

These unresolved issues will need to be taken into consideration particularly as CGM systems are considered for use for automated insulin dosing decisions. To date, through all these, overall accuracy of CGM systems has improved toward the proposed mark based on modeling to be accurate enough for making insulin dosing decisions in a MARD of less than $10 \% .^{20,21}$ Point accuracy is closely related to the problem of egregious errors, prolonged periods of substantial inaccuracy, which would lead to substantial insulin dosing errors in the setting of a closed-loop system. Indeed, this was a problem with previous CGM generations. ${ }^{22}$ For most devices, the distribution of sensor errors yields a curvilinear relationship between MARD and percentage large sensor deviations ( $>20 \%$ error), which defines a sweet spot of MARD $<10 \%$ and large deviations $<12 \%$ (Fig. 1). From in silico data, it seems that within the sweet spot further improvement of sensor accuracy leads to minimal gains in terms of clinical outcome improvement. $^{20,21} \mathrm{We}$ can, therefore, conclude that within these sensor accuracy limits, a CGM system should perform safely and efficiently, provided that CGM reliability and signal stability requirements are in place.

\section{Nonadjunctive Use of CGM}

Related to the question whether CGM is accurate enough for reliable AID systems is the question whether insulin dosing decisions by patients can be based on CGM alone, socalled nonadjunctive use of CGM. In September 2015, Dexcom (San Diego, CA) received approval for nonadjunct use of CGM in the EU (G5 Mobile CGM), followed by Food and Drug Administration (FDA) approval in December 2016. These approvals opened the regulatory path toward insulin dosing from sensor values, without a confirmatory fingerstick blood glucose. Approval of the inverse scenario-insulin shut-off based on sensor reading-was given with the approval of the Medtronic insulin pump that automatically shuts off insulin delivery at low glucose levels based on CGM data. If the patient is unresponsive to the CGM hypoglycemia alarm, insulin delivery is shut off for $2 \mathrm{~h}$ or until the patient restarts insulin delivery with the so-called low-glucose suspend feature. A pump with a predictive algorithm (predictive low-glucose suspend) was approved in the EU, which suspends insulin infusion when glucose is expected to fall below $80 \mathrm{mg} / \mathrm{dL}$ within the next $30 \mathrm{~min}$, for a maximum of $2 \mathrm{~h}$ or until glucose returns to above $70 \mathrm{mg} / \mathrm{dL} .^{23-25}$

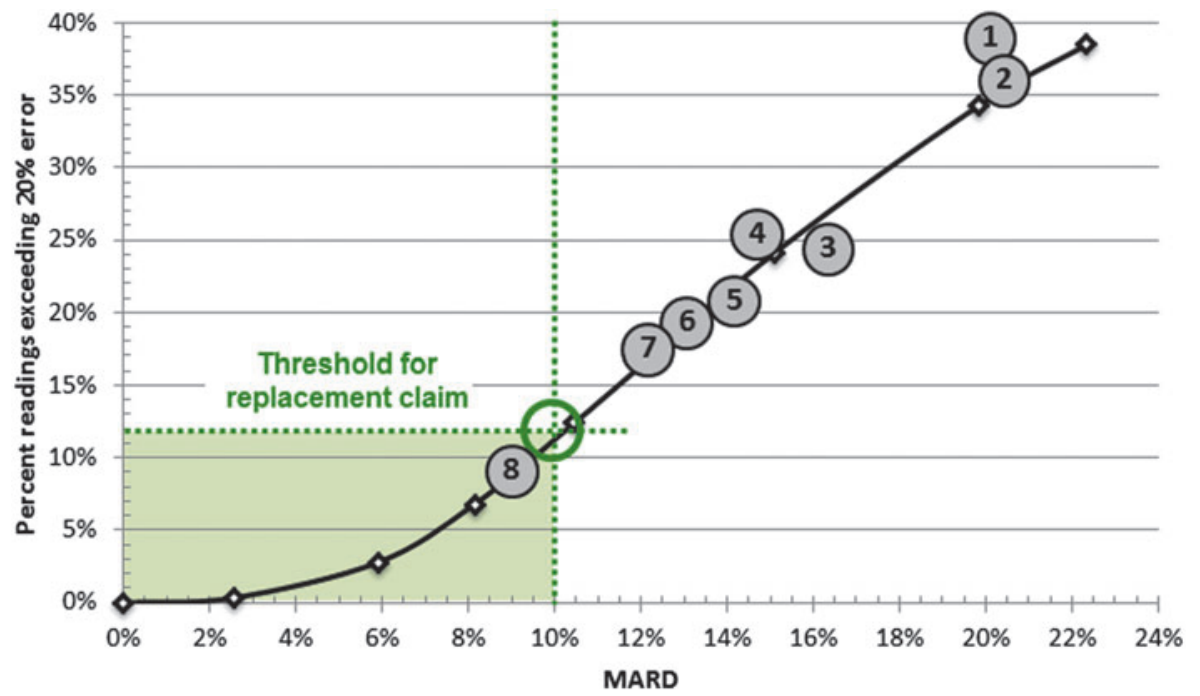

FIG. 1. There exists a curvilinear relationship between MARD and the frequency of large deviations (>20\%). Analyses of CGM accuracy data done by multiple studies show that this curve holds for a variety of sensors and is invariant across sensor manufacturers. Extensive in silico studies indicate that, within the rectangular area defined by MARD $<10 \%$ and large deviations $<12 \%$, further improvement in sensor accuracy does not contribute substantively to better glycemic outcomes. Thus, a sensor with accuracy within this rectangular area can be used as replacement for blood glucose meters. ${ }^{21}$ The sensors depicted in the figure include: (1) Medtronic Paradigm with Enlite sensor ${ }^{64}$; (2) Medtronic Paradigm with Enlite sensor ${ }^{7}$; (3) Animas Vibe with Dexcom G4 version A $^{64}$; (4) Freestyle Navigator I ${ }^{64}$; (5) Freestyle Navigator II ${ }^{65}$; (6) Dexcom G4 Platinum ${ }^{66}$; (7) Dexcom G4 Platinum ${ }^{7}$; (8) Dexcom G5 with software 505. ${ }^{67}$ CGM, continuous glucose monitoring; MARD, mean absolute relative difference. 


\section{Novel CGM Systems}

Recently, two innovative approaches have come to the market: flash glucose monitoring and implantable CGM. Flashglucose monitoring (Abbott Diabetes Care, Alameda) represents a new "on-demand" application of subcutaneous glucose sensing technology, effectively using CGM technology for replacement of SMBG and has been approved for nonadjunctive use in Europe. ${ }^{26,27}$ Flash-glucose monitoring could be regarded as CGM on demand, as a glucose value is only obtained when the sensor is interrogated by the handheld receiver. While this technology is not suitable to drive real-time CLC algorithms, it may be providing the perfect input signal for decision-support applications, which require user interaction for insulin bolusing and treatment-related actions. Its 2-week longevity and factory calibration increase its ease of use. In a study of adults with well-controlled type 1 diabetes, use of flash sensor-based glucose monitoring reduced time spent in hypoglycemia by a mean of $1.24 \mathrm{~h}$ per day. ${ }^{27}$ Patient enthusiasm and sales in Europe are notable, while the system is still under review at FDA.

Long-term implantable sensor technology has also recently entered the European market, and is also under review at FDA (Eversense ${ }^{\mathrm{TM}}$; Senseonics, Inc., Maryland). ${ }^{28}$ Implantable CGM increases patient friendliness, since frequent transcutaneous sensor insertions are replaced by a one-time insertion approximately every 3-6 months as a small surgical procedure doable for any trained healthcare provider. The transmitter can be removed easily, and the adhesive can be replaced daily after, for example, a shower. This CGM provides real-time information and should be easily incorporated into a closed-loop system, which would increase time in closed loop as the warm-up times inherent to transcutaneous sensor replacements are abolished. This is compared with currently available CGM systems, which have a $2 \mathrm{~h}$ window during which no values are shown while the signal stabilizes. First reported accuracy mentions a MARD of $11.1 \%$, and with improved technology the most recent MARD result is $8.8 \%$, according to a press announcement. Thus, this implantable device would fall within the accuracy limits discussed above. Additionally implantable CGM appears to not be susceptible to pressure-induced signal attenuation. ${ }^{29}$

\section{Automated Insulin Delivery}

The first wearable AID system-the Diabetes Assistant (DiAs) was introduced in late 2011 and initially tested in simultaneous outpatient studies in Italy and France. ${ }^{30}$ The DiAs was subsequently used in a randomized clinical trial published in $2014^{31}$ and in long-term trials of 24/7 AID. ${ }^{32-34}$ Since 2011, the number of published studies on this topic increased exponentially. ${ }^{35}$ Overnight insulin automation was the first target for many research groups, given the high potential benefit of reducing nocturnal hypoglycemia without the complicating issue of meals. The ability to reduce hypoglycemia at night was proven out by groups, including Nimri et al. in a study comparing AID to sensor-augmented pump (SAP) therapy overnight for 6 weeks. ${ }^{36}$ Kropff et al. expanded the use of insulin automation by starting at dinnertime with use of the system until the next morning over a period of 8 weeks, publishing the first study to demonstrate improvement in A1C as compared with SAP (difference of $-0.2 \%$ ) in addition to a reduction in time spent in hyperglycemia and hypoglycemia. ${ }^{34}$ In 2015 , Thabit et al. published the use of AID in 33 adults and 25 children and adolescents, which improved time in euglycemia, reduced time in hypoglycemia, and reduced hemoglobin $\mathrm{A} 1 \mathrm{C}$ (difference of $-0.3 \%$ ) compared with SAP. ${ }^{37}$ Adults used the AP system day and night for 12 weeks, whereas children and adolescents used the system overnight for 12 weeks.

With the maturation of AID, companies are bringing AID systems to market. In 2016, the FDA approved the first hybrid system that automates basal insulin delivery in response to hyperglycemia and hypoglycemia, the Medtronic 670G. The pump basal rate is automated based on a proportional-integral-derivative algorithm with IOB feedback. ${ }^{38}$ This system still depends on the user to enter postmeal insulin corrections, meal and exercise information. In a pivotal trial of 94 adults and 30 adolescents with type 1 diabetes, use of the $670 \mathrm{G}$ improved $\mathrm{A} 1 \mathrm{C}$ by $0.5 \%$ and reduced time in hypoglycemia by $44 \%$ as compared with baseline. ${ }^{39}$ It should be noted that this study was designed to evaluate safety and was not a randomized controlled trial. The comparison was A1C before the intervention and CGM values during a 2-week runin period without AID. The next step in this line of development would be commercial AID systems that automate postmeal correction boluses. ${ }^{40}$ Long-term home-based clinical trials of such systems are under way.

\section{Multihormonal Systems}

The normal human pancreas secretes glucagon to defend against hypoglycemia, a system which is dysfunctional in type 1 diabetes. ${ }^{41}$ The addition of glucagon to AID is attractive to counteract the slow offset of subcutaneous insulin, but adds to overall system complexity and cost. ${ }^{42}$ Multiple academic groups have demonstrated the short-term efficacy and safety of automated insulin and glucagon delivery in people with type 1 diabetes. ${ }^{43-46}$ Beta Bionics in the United States and Inreda Diabetic B.V. in the Netherlands are working toward commercializing the first bihormonal systems and are anticipated to launch clinical trials in 2017. The absence of a glucagon approved for pump use remains a barrier to commercialization of a dual-hormone system, but is being addressed by companies, including Zealand and Xeris pharmaceuticals, ${ }^{47}$ both of which have stable glucagon formulations in clinical testing. Nevertheless, the future of dual insulin+glucagon control is uncertain. Longer clinical trials are still needed to support the long-term efficacy and safety of chronic glucagon administration as well as to determine if the benefits outweigh the complexity of an insulin and glucagon system.

Another potential multihormonal approach involves the use of synthetic amylin. Amylin is cosecreted with insulin from healthy beta cells in response to meals, and is therefore absent in type 1 diabetes. Thus, hormone replacement therapies should consider the use of amylin as well. Amylin's synthetic analogue, pramlintide, is an approved adjunctive therapeutic option for management of type 1 diabetes. Studies have demonstrated that pramlintide inhibits glucagon concentrations, delays peak meal rate of appearance, lowers postprandial glucose excursions, and improves insulin sensitivity in healthy subjects. ${ }^{48,49}$ CLC, using adjunctive pramlintide, has previously been tested. ${ }^{50}$ An in silico model has been designed to determine the optimal ratio for coformulation of insulin and pramlintide, ${ }^{51}$ and the in silico results were further confirmed by the PICOS study. ${ }^{52}$ Ongoing clinical trials now pursue this multihormonal approach as an alternative to insulin+glucagon closed loop. 


\section{The Challenge of Exercise and Multisignal AID}

AID will certainly revolutionize the treatment of T1D, but replicating the effectiveness of the normal human pancreas meets significant challenges. Exercise has proven to be a particularly difficult-to-control metabolic disturbance. Exercise increases muscle demand for glucose and can increase insulin sensitivity for hours later. ${ }^{53}$ Compared with devices of a few years ago, modern CGM technology has a significantly shorter delay and improved accuracy in the hypoglycemic range. ${ }^{7,8,54,55}$ These advances allow for earlier detection of glucose drops during exercise. But given the long-time delays of insulin action, stopping insulin delivery for a rapidly falling glucose is unlikely to prevent hypoglycemia.

Ideally patients would announce exercise $90 \mathrm{~min}$ in advance of exercise to reduce the risk of hypoglycemia, ${ }^{56}$ but advanced announcement is unrealistic in many cases. Automated glucagon delivery combined with exercise announcement has been shown to reduce hypoglycemia. ${ }^{57}$ Ideally, a system would be able to automatically detect exercise. Much work has been done to detect exercise through the use of heart rate alone $^{58,59}$ or in combination with accelerometry. ${ }^{60}$ Because these additional signals detect physical activity faster than CGM alone, the use of automated exercise detection using these signals may prove to be an essential part of AP systems to reduce exercise-related hypoglycemia. ${ }^{61}$

\section{Embedded and Mobile AID Systems}

Traditionally, it is assumed that the CLC algorithm would be embedded into the insulin pump. The pump would, therefore, run a control algorithm, user interface, and all device communications. New devices, such as the Medtronic 670G discussed above are already approved to carry out these functions. This embedded approach has several advantages, including short signal path and potentially greater reliability. However, embedding several computationally demanding functions into an insulin pump may be taking the pump out of the realm of its core design. This may limit the rate of deployment of technology advancements, particularly features such as an attractive user interface or Cloud communication.

Adding new functions also increases greatly the complexity of the pump, and thus the development time of a device that must be absolutely robust and resilient to interference. An alternative is a Mobile AID configuration using consumer electronics (e.g., a smart phone) as an outboard closed-loop controller. This solution has a number of advantages as well: (i) smart phones are inexpensive, available, computationally capable of running CLC algorithms, and wirelessly connectable to various devices and data networks-no current insulin pump offers similar capabilities; (ii) the life cycle of a smart phone is months, as opposed to years for insulin pumps; thus, smart phones allow easier updates of user interface and device form factor; (iii) psychologists share that patients, particularly youth, miss boluses because of reluctance to use their insulin pump in public; however, no one is reluctant to use a phone and that may be a key to AID success and widespread adoption. The last remaining question is whether a modular system based on consumer electronics could receive regulatory clearance. A positive development in this direction was the recent FDA approval of the International Closed-Loop Trial as a pivotal trial of a mobile AID system.

\section{Conclusion}

Enabled by CGM advances, AID is evolving to become the standard of care for managing type 1 diabetes. Insulin delivery automation is a major advance, but will not approximate the very tight glucose range seen in people without diabetes. It will be important for healthcare providers and patients alike to understand the limits of insulin delivered subcutaneously. The development of ultra-rapid-acting insulin analogues is expected to bring the technology one further step closer to physiological glucose homeostasis. ${ }^{62}$ Insulin delivery automation is dependent on CGM accuracy and there remain additional opportunities to improve upon CGM technology. These would include even better point accuracy and less egregious errors, prolonged sensor lifetime, but also a solution for acetaminophen and other interferences. Future CGM devices are likely to be able to address most, if not all, of these issues.

The automation of insulin delivery will be further supported by the introduction of CLC algorithms that are capable of utilizing multiple signals reflecting better the physiological state of a person than CGM alone. Extensive studies of multihormonal systems will gauge the viability of insulin+glucagon control, and of alternatives such as insulin+pramlintide coadministration or coformulation. All of these systems will benefit from the emerging understanding that individualization and real-time adaptation is necessary to account for the rapidly changing insulin requirements of a person with diabetes.

Individualization of the control algorithm refers to tuning its initial parameters to the specifics of each individual, a "prescription" for the CLC algorithm that is typically done using a person's body weight and insulin requirements (e.g., carbohydrate ratio, correction factor, and basal rate). Adaptation of the algorithm refers to tracking of biobehavioral events and profiles and real-time adjustment of CLC parameters in response. As the system is continually subjected to external challenges and metabolic perturbations, tracking patient state and adjustment of key control parameters become imperative. Engineering methods, such as "run-to-run control" and other techniques, are at various stages of development to address these issues. ${ }^{5}$ It remains unknown what role healthcare providers will take in adjusting settings for advanced CLC systems. Clearly, providers, diabetes educators, and patients will need to be trained on the use of these advance systems.

The form factor of upcoming AID systems would include Embedded or Mobile approaches, or a combination of the two. The current trend is toward all-in-one solutions embedded in the insulin pump. In the near future this trend may be expanded by adoption of mobile systems based on consumer electronics. While it is well understood that cybersecurity issues need to be resolved before an ordinary smart phone can run an AID app, the proposition is tempting to have a "universal" mobile system that can connect to any sufficiently capable sensor or insulin pump and close the loop on demand. Steps in this direction are done by the new cybersecurity standard for connected diabetes devices $^{63}$ and by sensors such as the Dexcom G5, which can operate independently from a CGM receiver, using direct connection with a smart phone.

\section{Author Disclosure Statement}

J.R.C. has a financial interest in Pacific Diabetes Technologies Inc, a company that may have a commercial interest in the results of this type of research and technology. In addition, 
J.R.C. reports research support from Dexcom and Tandem Diabetes Care outside the submitted work. The time for J.R.C. to prepare this article was supported by grant 1DP3DK10104401 from NIH/NIDDK. This potential conflict of interest has been reviewed and managed by OHSU. J.H.D.V. reports personal fees from Roche and research support from Abbott, Dexcom, Medtronic, Sanofi, and Senseonics. B.K. reports grants from Sanofi-Aventis, personal fees from Dexcom and Sanofi-Aventis; support from Dexcom, Roche Diagnostics Inc., and Tandem Diabetes Care outside the submitted work. In addition, B.K. has patent \# 8,562,587, October 22, 2013, with royalties paid to Animas Corporation, and is Board member and shareholder in TypeZero Technologies.

\section{References}

1. Ruan Y, Thabit H, Leelarathna L, et al.: Variability of insulin requirements over 12 weeks of closed-loop insulin delivery in adults with type 1 diabetes. Diabetes Care 2016;39:830-832.

2. Hovorka R: Continuous glucose monitoring and closedloop systems. Diabet Med 2006;23:1-12.

3. Steil GM, Panteleon AE, Rebrin K: Closed-loop insulin delivery-the path to physiological glucose control. Adv Drug Deliv Rev 2004;56:125-144.

4. Cobelli C, Man CD, Sparacino G, et al.: Diabetes: models, signals, and control. IEEE Rev Biomed Eng 2009;2:54-96.

5. Doyle FJ, 3rd, Huyett LM, Lee JB, et al.: Closed-loop artificial pancreas systems: engineering the algorithms. Diabetes Care 2014;37:1191-1197.

6. Hovorka R: Closed-loop insulin delivery: from bench to clinical practice. Nat Rev Endocrinol 2011;7:385-395.

7. Kropff J, Bruttomesso D, Doll W, et al.: Accuracy of two continuous glucose monitoring systems: a head-to-head comparison under clinical research centre and daily life conditions. Diabetes Obes Metab 2015;17:343-349.

8. Wentholt IM, Vollebregt MA, Hart AA, et al.: Comparison of a needle-type and a microdialysis continuous glucose monitor in type 1 diabetic patients. Diabetes Care 2005;28:2871-2876.

9. Fachinetti A, Sparacino G, Guerra S, et al.: Real-time improvement of continuous glucose monitoring accuracy; the smart sensor concept. Diabetes Care 2013;26:793-800.

10. Klueh U, Liu Z, Feldman B, et al.: Metabolic biofouling of glucose sensors in vivo: role of tissue microhemorrhages. $\mathrm{J}$ Diabetes Sci Technol 2011;5:583-595.

11. Klueh U, Qiao Y, Czajkowski C, et al.: Basement membranebased glucose sensor coatings enhance continuous glucose monitoring in vivo. J Diabetes Sci Technol 2015;9:957-965.

12. CLSI: Performance metrics for continuous interstitial glucose monitoring; approved guideline. CLSI document POCT05-A. Wayne, PA: Clinical and Laboratory Standards Institute; 2008.

13. Clarke WL: The original Clarke Error Grid Analysis (EGA). Diabetes Technol Ther 2005;7:776-779.

14. Kovatchev BP, Gonder-Frederick LA, Cox DJ, et al.: Evaluating the accuracy of continuous glucose-monitoring sensors: continuous glucose-error grid analysis illustrated by TheraSense Freestyle Navigator data. Diabetes Care 2004;27:1922-1928.

15. Pfutzner A, Klonoff DC, Pardo S, et al.: Technical aspects of the Parkes error grid. J Diabetes Sci Technol 2013;7:1275-1281.

16. Klonoff DC, Lias C, Vigersky R, et al.: The surveillance error grid. J Diabetes Sci Technol 2014;8:658-672.

17. Andelin M, Kropff J, Matuleviciene V, et al.: Assessing the accuracy of continuous glucose monitoring (CGM) calibrated with capillary values using capillary or venous glucose levels as a reference. J Diabetes Sci Technol 2016;10:876-884.

18. Castle JR, Ward WK: Amperometric glucose sensors: sources of error and potential benefit of redundancy. J Diabetes Sci Technol 2010;4:221-225.

19. Bequette BW: Challenges and recent progress in the development of a closed-loop artificial pancreas. Annu Rev Control 2012;36:255-266.

20. Kovatchev BP: Hypoglycemia reduction and accuracy of continuous glucose monitoring. Diabetes Technol Ther 2015;17:530-533.

21. Kovatchev BP, Patek SD, Ortiz EA, et al.: Assessing sensor accuracy for non-adjunct use of continuous glucose monitoring. Diabetes Technol Ther 2015;17:177-186.

22. Leelarathna L, Nodale M, Allen JM, et al.: Evaluating the accuracy and large inaccuracy of two continuous glucose monitoring systems. Diabetes Technol Ther 2013;15:143-149.

23. Maahs DM, Calhoun P, Buckingham BA, et al.: A randomized trial of a home system to reduce nocturnal hypoglycemia in type 1 diabetes. Diabetes Care 2014;37:1885-1891.

24. Buckingham BA, Cameron F, Calhoun P, et al.: Outpatient safety assessment of an in-home predictive low-glucose suspend system with type 1 diabetes subjects at elevated risk of nocturnal hypoglycemia. Diabetes Technol Ther 2013;15:622-627.

25. The effect of intensive treatment of diabetes on the development and progression of long-term complications in insulin-dependent diabetes mellitus. The Diabetes Control and Complications Trial Research Group. N Engl J Med 1993;329:977-986.

26. Bailey T, Bode BW, Christiansen MP, et al.: The performance and usability of a factory-calibrated flash glucose monitoring system. Diabetes Technol Ther 2015;17:787-794.

27. Bolinder J, Antuna R, Geelhoed-Duijvestijn P, et al.: Novel glucose-sensing technology and hypoglycaemia in type 1 diabetes: a multicentre, non-masked, randomised controlled trial. Lancet 2016;388:2254-2263.

28. Kropff J, Choudhary P, Neupane S, et al.: Accuracy and longevity of an implantable continuous glucose sensor in the PRECISE Study: a 180-day, prospective, multicenter, pivotal trial. Diabetes Care 2017;40:63-68.

29. Wang X, Ioacara S, DeHennis A: Long-term home study on nocturnal hypoglycemic alarms using a new fully implantable continuous glucose monitoring system in type 1 diabetes. Diabetes Technol Ther 2015;17:780-786.

30. Cobelli C, Renard E, Kovatchev BP, et al.: Pilot studies of wearable outpatient artificial pancreas in type 1 diabetes. Diabetes Care 2012;35:e65-e67.

31. Kovatchev BP, Renard E, Cobelli C, et al.: Safety of outpatient closed-loop control: first randomized crossover trials of a wearable artificial pancreas. Diabetes Care 2014;37:1789-1796.

32. Anderson SM, Raghinaru D, Pinsker JE, et al.: Multinational home use of closed-loop control is safe and effective. Diabetes Care 2016;39:1143-1150.

33. Kovatchev B, Cheng P, Anderson SM, et al.: Feasibility of long-term closed-loop control: a multicenter 6-month trial of 24/7 automated insulin delivery. Diabetes Technol Ther 2017;19:18-24.

34. Kropff J, Del Favero S, Place J, et al.: 2 month evening and night closed-loop glucose control in patients with type 1 diabetes under free-living conditions: a randomised crossover trial. Lancet Diabetes Endocrinol 2015;3:939-947.

35. Thabit H, Hovorka R: Coming of age: the artificial pancreas for type 1 diabetes. Diabetologia 2016;59:1795-1805. 
36. Nimri R, Muller I, Atlas E, et al.: MD-Logic overnight control for 6 weeks of home use in patients with type 1 diabetes: randomized crossover trial. Diabetes Care 2014;37:3025-3032.

37. Thabit H, Tauschmann M, Allen JM, et al.: Home use of an artificial beta cell in type 1 diabetes. N Engl J Med 2015; 373:2129-2140.

38. Ruiz JL, Sherr JL, Cengiz E, et al.: Effect of insulin feedback on closed-loop glucose control: a crossover study. J Diabetes Sci Technol 2012;6:1123-1130.

39. Bergenstal RM, Garg S, Weinzimer SA, et al.: Safety of a hybrid closed-loop insulin delivery system in patients with type 1 diabetes. JAMA 2016;316:1407-1408.

40. DeVries JH: The artificial pancreas-ready for prime time? Lancet Diabetes Endocrinol 2017;5:238-239.

41. Dagogo-Jack SE, Craft S, Cryer PE: Hypoglycemiaassociated autonomic failure in insulin-dependent diabetes mellitus. Recent antecedent hypoglycemia reduces autonomic responses to, symptoms of, and defense against subsequent hypoglycemia. J Clin Invest 1993;91:819-828.

42. Castle JR: Is glucagon needed in type 1 diabetes? Lancet Diabetes Endocrinol 2015;3:578-579.

43. Castle JR, Engle JM, El Youssef J, et al.: Novel use of glucagon in a closed-loop system for prevention of hypoglycemia in type 1 diabetes. Diabetes Care 2010;33:1282-1287.

44. Russell SJ, El-Khatib FH, Sinha M, et al.: Outpatient glycemic control with a bionic pancreas in type 1 diabetes. $\mathrm{N}$ Engl J Med 2014;371:313-325.

45. Haidar A, Legault L, Messier V, et al.: Comparison of dualhormone artificial pancreas, single-hormone artificial pancreas, and conventional insulin pump therapy for glycaemic control in patients with type 1 diabetes: an open-label randomised controlled crossover trial. Lancet Diabetes Endocrinol 2015;3:17-26.

46. Blauw H, van Bon AC, Koops R, et al.: Performance and safety of an integrated bihormonal artificial pancreas for fully automated glucose control at home. Diabetes Obes Metab 2016;18:671-677.

47. Castle JR, Youssef JE, Branigan D, et al.: Comparative pharmacokinetic/pharmacodynamic study of liquid stable glucagon versus lyophilized glucagon in type 1 diabetes subjects. J Diabetes Sci Technol 2016;10:1101-1107.

48. Hinshaw L, Schiavon M, Mallad A, et al.: Effects of delayed gastric emptying on postprandial glucose kinetics, insulin sensitivity, and beta-cell function. Am J Physiol Endocrinol Metab 2014;307:E494-E502.

49. Woerle HJ, Albrecht M, Linke R, et al.: Importance of changes in gastric emptying for postprandial plasma glucose fluxes in healthy humans. Am J Physiol Endocrinol Metab 2008;294:E103-E109.

50. Weinzimer SA, Sherr JL, Cengiz E, et al.: Effect of pramlintide on prandial glycemic excursions during closedloop control in adolescents and young adults with type 1 diabetes. Diabetes Care 2012;35:1994-1999.

51. Micheletto F, Dalla Man C, Kolterman O, et al.: In silico design of optimal ratio for co-administration of pramlintide and insulin in type 1 diabetes. Diabetes Technol Ther 2013;15:802-809.

52. Riddle MC, Yuen KC, de Bruin TW, et al.: Fixed ratio dosing of pramlintide with regular insulin before a standard meal in patients with type 1 diabetes. Diabetes Obes Metab 2015;17:904-907.

53. McMahon SK, Ferreira LD, Ratnam N, et al.: Glucose requirements to maintain euglycemia after moderate-intensity afternoon exercise in adolescents with type 1 diabetes are increased in a biphasic manner. J Clin Endocrinol Metab 2007;92:963-968.

54. Garcia A, Rack-Gomer AL, Bhavaraju NC, et al.: Dexcom G4AP: an advanced continuous glucose monitor for the artificial pancreas. J Diabetes Sci Technol 2013;7:1436-1445.

55. Keenan DB, Mastrototaro JJ, Zisser H, et al.: Accuracy of the Enlite 6-day glucose sensor with guardian and Veo calibration algorithms. Diabetes Technol Ther 2012;14:225-231.

56. Schiavon M, Dalla Man C, Kudva YC, et al.: In silico optimization of basal insulin infusion rate during exercise: implication for artificial pancreas. J Diabetes Sci Technol 2013;7:1461-1469.

57. Taleb N, Emami A, Suppere C, et al.: Efficacy of singlehormone and dual-hormone artificial pancreas during continuous and interval exercise in adult patients with type 1 diabetes: randomised controlled crossover trial. Diabetologia 2016;59:2561-2571.

58. Breton MD, Brown SA, Karvetski $\mathrm{CH}$, et al.: Adding heart rate signal to a control-to-range artificial pancreas system improves the protection against hypoglycemia during exercise in type 1 diabetes. Diabetes Technol Ther 2014;16:506-511.

59. DeBoer MD, Chernavvsky DR, Topchyan K, et al.: Heart rate informed artificial pancreas system enhances glycemic control during exercise in adolescents with T1D. Pediatr Diabetes 2016.

60. Jacobs PG, Resalat N, El Youssef J, et al.: Incorporating an exercise detection, grading, and hormone dosing algorithm into the artificial pancreas using accelerometry and heart rate. J Diabetes Sci Technol 2015;9:1175-1184.

61. Jacobs PG, El Youssef J, Reddy R, et al.: Randomized trial of a dual-hormone artificial pancreas with dosing adjustment during exercise compared with no adjustment and sensor-augmented pump therapy. Diabetes Obes Metab 2016;18:1110-1119.

62. Heinemann L, Muchmore D: Coverage of prandial insulin requirements: an elusive goal. Diabetes Technol Ther 2017;19:7-8.

63. Diabetes Technology Society: Standard for wireless disbetes device security. May 23, 2016. www.diabetestechnology .org/dtsec-standard-final.pdf (accessed March 28, 2017).

64. Luijf YM, Mader JK, Doll W, et al.: Accuracy and reliability of continuous glucose monitoring systems: a head-to-head comparison. Diabetes Technol Ther 2013;15:722-727.

65. Thabit H, Leelarathna L, Wilinska ME, et al.: Accuracy of continuous glucose monitoring during three closed-loop home studies under free-living conditions. Diabetes Technol Ther 2015;17:801-807.

66. Christiansen M, Bailey T, Watkins E, et al.: A new-generation continuous glucose monitoring system: improved accuracy and reliability compared with a previous-generation system. Diabetes Technol Ther 2013;15:881-888.

67. Peyser TA, Nakamura K, Price D, et al.: Hypoglycemic accuracy and improved low glucose alerts of the latest dexcom G4 platinum continuous glucose monitoring system. Diabetes Technol Ther 2015;17:548-554.

Address correspondence to: Jessica R. Castle, MD

Division of Endocrinology

Harold Schnitzer Diabetes Health Center Oregon Health \& Science University 3181 SW Sam Jackson Park Road Portland, OR 97239

E-mail: castleje@ohsu.edu 